\title{
Efficacy and safety of EUS-guided gastroenterostomy for benign and malignant gastric outlet obstruction: a systematic review and meta-analysis
}

\section{(ㄷ)(i) $\Theta$}

\author{
Authors
}

Thomas R. McCarty1,2, Rajat Garg ${ }^{3}$, Christopher C. Thompson ${ }^{1,2}$, Tarun Rustagi ${ }^{4}$

Institutions

1 Division of Gastroenterology, Hepatology and Endoscopy, Brigham and Women's Hospital, Boston, Massachusetts, United States

2 Harvard Medical School, Boston, Massachusetts, United States

3 Cleveland Clinic, Cleveland, Ohio, United States

4 Division of Gastroenterology and Hepatology, University of New Mexico, Albuquerque, New Mexico, United States

submitted 12.6.2019

accepted after revision 31.7.2019

\author{
Bibliography \\ DOI https://doi.org/10.1055/a-0996-8178 | \\ Endoscopy International Open 2019; 07: E1474-E1482 \\ (c) Georg Thieme Verlag KG Stuttgart · New York \\ eISSN 2196-9736
}

Corresponding author

Tarun Rustagi, MD, Division of Gastroenterology and Hepatology, University of New Mexico, MSC10 5550, 1 University of New Mexico, Albuquerque NM 87131

Fax: +1-505-272-9751

tarunrustagi06@gmail.com

丹 Supplementary material

Online content viewable at:

https://doi.org/10.1055/a-0996-8178

\section{ABSTRACT}

Background and study aims Endoscopic ultrasoundguided gastroenterostomy (EUS-GE) is a novel endoscopic procedure designed to facilitate sustained luminal patency in patients with gastric outlet obstruction. The primary aim of this study was to evaluate the efficacy and safety of EUSGE for treatment of gastric outlet obstruction.

Methods Searches of PubMed, EMBASE, Web of Science, and Cochrane Library databases were performed through April 2019. Patients with benign and malignant gastric outlet obstruction were included. Measured outcomes included: immediate technical and clinical success as well as rate of serious adverse events (AEs). Heterogeneity was assessed with Cochran $Q$ test and $R^{2}$ statistics. Publication bias was ascertained by funnel plot and Egger regression testing.

Results A total of five studies ( $n=199$ patients; $45.73 \%$ male) were included in this study. Four retrospective studies and one prospective study were analyzed. Mean age of patients that underwent the EUS-GE procedure was 64.52 \pm 1.37 years with a pooled mean follow-up period of $4.32 \pm$ 1.65 months. In $21 \%$ of patients $(n=43)$, gastric outlet obstruction was due to benign causes. Immediate technical success was $92.90 \%\left(95 \% \mathrm{Cl} ; 88.26-95.79 ; \mathrm{I}^{2}=0.00 \%\right)$ and reported in all studies. The clinical success rate of EUS-GE was $90.11 \%\left(95 \% \mathrm{Cl} ; 84.64-93.44 ; I^{2}=0.00 \%\right)$. Serious AEs occurred in $5.61 \%\left(95 \% \mathrm{Cl} ; 2.87-10.67 ; \mathrm{I}^{2}=1.67 \%\right)$ of cases and were related to peritonitis, perforation, bleeding, and abdominal pain. Re-intervention rate was $11.43 \%$ (95\% Cl; $\left.7.29-17.46 ; I^{2}=17.38 \%\right)$.

Conclusions EUS-GE appears to provide an effective and safe minimally invasive alternative for treatment of benign and malignant gastric outlet obstruction.

\section{Introduction}

Gastric outlet obstruction is a clinical condition characterized by nausea and vomiting, postprandial fullness, as well as epigastric abdominal pain and weight loss as a pathophysiologic consequence of a mechanical obstruction within the gastrointestinal tract. Previously termed pyloric stenosis, gastric outlet obstruction is the preferred or standard terminology as mechanical obstruction may occur in the distal stomach, pylorus, or duodenum. The condition itself is typically classified based upon etiology with benign and malignant causes. Although the primary etiology over time has changed from peptic ulcer disease and other benign entities to a more common association with underlying malignancy, gastric outlet obstruction has 
a significant effect on patient morbidity and overall quality of life [1 - 5].

Traditionally, surgical gastrojejunostomy has been the primary treatment for both benign and malignant gastric outlet obstruction although the procedure itself is associated with a high complication rate that approaches more than $40 \%[6,7]$. Although surgical gastrojejunostomy is currently the standard of care for palliative treatment, the procedure is further limited by prolonged recovery times delaying chemotherapy for malignancy-related obstructions, delayed gastric emptying and gastroparesis, as well as substantial procedure-associated costs [7]. Given the significant morbidity of this surgical procedure, less-invasive endoscopic alternatives have been developed including endoscopic ultrasound-guided gastroenterostomy (EUS-GE).

EUS-GE has recently emerged as a procedure to treat patients with gastric outlet obstruction as an alternative to surgery or to standard endoscopy when endoscopic enteral stent placement is not possible [7,8]. Although EUS-GE was first described by Fritscher-Ravens et al. in the early 2000 s, it was only after the recent availability of a bi-flanged lumen-apposing metal stent (LAMS) that EUS-GE was clinically adopted. EUS-GE performed with placement of a LAMS has emerged as another treatment option that may provide long-term luminal patency without associated morbidity and complication rates of a surgical approach $[9,10]$. While this novel procedure is designed to facilitate sustained luminal patency in patients with gastric outlet obstruction while avoiding the morbidity of a surgical procedure, the efficacy and safety is EUS-GE remains less clear.

The primary aim of this study was to perform a systematic review and meta-analysis to evaluate the efficacy and safety of EUS-GE for treatment of benign and malignant gastric outlet obstruction.

\section{Methods}

\section{Literature search}

A comprehensive search of the literature was performed to identify articles that examined the EUS-GE procedure. Systematic searches of PubMed, EMBASE, Web of Science, and the Cochrane Library databases were performed from 2001 through April 1, 2019. The following search terms included: "endoscopic ultrasound-guided gastroenterostomy (EUS-GE)," "endoscopic ultrasound-guided gastrojejunostomy (EUS-GJ)," "endoscopic ultrasound-guided gastroduodenostomy (EUSGD)," "endoscopic ultrasound and gastric outlet obstruction," and "endoscopic ultrasound and pyloric obstruction." All relevant articles irrespective of year of publication, type of publication, or publication status were included. Titles and abstracts of all potentially relevant studies were screened for eligibility. The reference lists of studies of interest were then manually reviewed for additional articles by cross-checking bibliographies. Two reviewers (TRM and RG) independently screened the titles and abstracts of all the articles according to predefined inclusion and exclusion criteria. Any differences were resolved by mutual agreement and in consultation with the third reviewer (TR). In the case of studies with incomplete information, con- tact was attempted with the principal authors to obtain additional data.

\section{Study selection criteria}

This study was prospectively submitted in PROSPERO, an international database of prospectively registered systematic reviews in health and social care. The Preferred Reporting Items for Systematic Reviews and Meta-Analyses (PRISMA) statement outline and Meta-Analysis of Observational Studies in Epidemiology (MOOSE) reporting guidelines for reporting systematic reviews and meta-analyses was used to report findings [11,12]. Only studies investigating use of the EUS-GE modality for treatment of gastric outlet obstruction were included. Only human subject studies were considered in the analysis. A study was excluded if deemed to have insufficient data, as were review articles, editorials, and correspondence letters that did not report independent data. Case series and reported studies with fewer than 10 patients were excluded. Multiple published work from similar authors was evaluated for overlapping enrollment times to preserve independence of observations. Participants included patients of any age in whom presence of gastric outlet obstruction was suspected or confirmed based upon prior imaging studies.

\section{Procedure characteristics}

All patients included in this study for analysis underwent EUSGE for treatment of gastric outlet obstruction. Multiple EUS-GE procedures (i. e., unassisted and assisted techniques) were also analyzed including: direct EUS-GE, balloon-assisted, EUS-guided double-balloon-occluded gastrojejunostomy bypass (EPASS), nasobiliary drain, and Natural Orifice Transluminal Endoscopic Surgery (NOTES). All sizes of available LAMS were included as well as both cautery-enhanced lumen-apposing metal stent (CE-LAMS) and non-cautery-enhanced lumen-apposing metal stent (NCE-LAMS). Both EUS-GJ and EUS-GD were included.

\section{Outcome measures}

The primary outcome measurement in this study was the feasibility, efficacy, and tolerability of EUS-GE in patients with gastric outlet obstruction. Efficacy and safety of the device were measured by immediate technical success rate (i.e., ability to perform the procedure without issue), clinical success as defined by individual study authors, and serious adverse events (AEs) reported during follow-up. Clinical success was also measured by level of oral intake or relief of symptoms (i. e., early satiety, nausea, vomiting) using the validated gastric outlet obstruction scoring system (GOOSS) [13]. This GOOSS is designed to provide an objective grade to determine patients' ability to eat before and after gastric outlet obstruction procedure (i.e., EUS-GE). Other measured outcomes included baseline patient characteristics (i.e., mean age, gender, benign or malignancy etiology of gastric out obstruction, and prior gastrointestinal treatment), procedural-related characteristics (i.e., EUS-GE technique, timing of procedure, and other AEs), and duration of follow-up. 


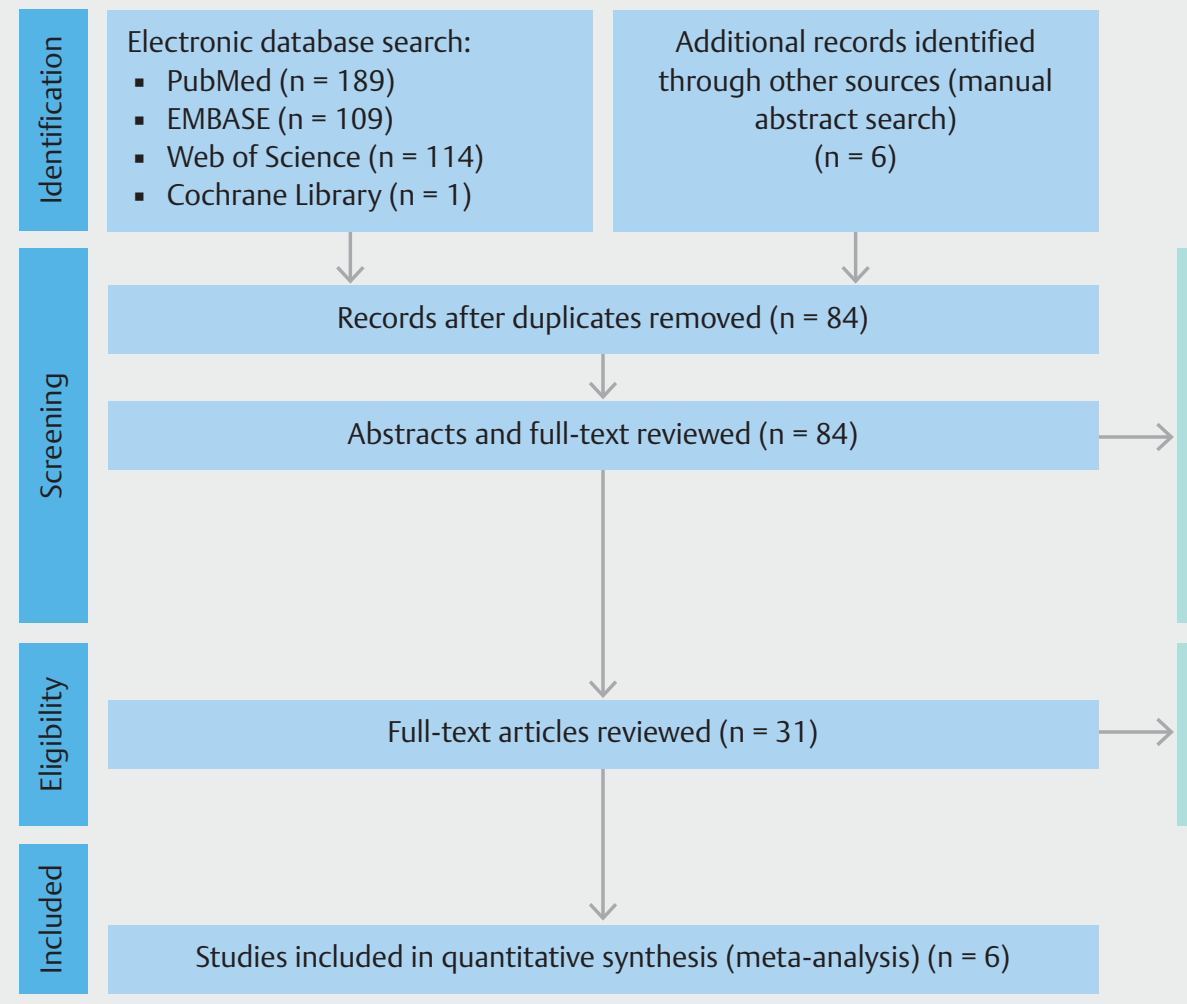

Excluded based on title and abstract review

$(n=53)$

- basic science articles, review articles, editorials

- observational studies

- reported non-effective interventions or interventions not used in clinical practice

Full-text articles excluded $(n=25)$

- Review/commentaries

- Insufficient data

- Follow-up of initial study

- Fig. 1 Preferred Reporting Items for Systematic Reviews and Meta-Analyses (PRISMA) flow chart of literature search results for EUS-guided gastroenterostomy for the treatment of gastric outlet obstruction.

\section{Statistical analysis}

This meta-analysis was performed by calculating pooled proportions. After appropriate studies were identified through systematic review, the individual study proportion was transformed into a quantity using the Freeman-Tukey variant of the arcsine square root transformed proportion. Then the pooled proportion was calculated as the back transform of the weighted mean of the transformed proportions, using inverse arcsine variance weights for the fixed effects model and DerSimonianLaird weights for the random effects model [14-17].

Measured outcomes comparing the pre- and post-procedure GOOSS were also obtained. From this, standardized mean difference was calculated and transformed to the natural logarithm before pooling, and the variance was calculated. Fixed-effects models were applied to pre- and post-EUS-GE data to determine effect size and corresponding $95 \%$ confidence intervals (Cls). Tabular and graphical analyses were performing using Comprehensive Meta-Analysis software, version 3 (BioStat, Englewood, New Jersey, United States). Additional analyses were performed with the aid of the Stata 13.0 software package (Stata Corp LP, College Station, Texas, United States).

\section{Risk of bias and quality assessment}

Risk of bias and quality of observational studies was evaluated using the Newcastle-Ottawa Quality Assessment Scale [18]. Domains assessed were selection of cohort, ascertainment of exposure/comparator, and assessment of outcome. Based upon this, criteria score $\geq 5$ was consistent with high quality, 3 to 4 medium quality, and $\leq 2$ was considered low quality. Quality of randomized clinical trials (RCTs) was assessed using the JADAD score [19]. Two authors (TRM and RG) independently extracted data and assessed risk of bias and study quality for each of the articles. Any disagreements were resolved by discussion and consensus, and in consultation with the third reviewer (TR).

\section{Investigations of heterogeneity}

Heterogeneity was assessed for the individual meta-analyses using the chi squared test and the 12 statistic [20]. Significant heterogeneity was defined as $P<0.05$ using the Cochran $Q$ test or $P^{2}>50 \%$, with values $>50 \%$ indicating substantial heterogeneity. To assess for publication bias, a funnel plot was created and visually inspected for asymmetry and quantitatively using Egger regression testing $[21,22]$.

\section{Results}

\section{Baseline study information and patient characteristics}

This meta-analysis included a total of five studies including years from 2016 to 2019 [7,23-26]. A PRISMA flow chart of search results is shown in $\mathbf{F i g . 1}$. Four studies were multicenter with two single-center trials also included. Four included 
studies were retrospective in nature with an additional prospective study analyzed. A total of 199 patients were included in this study. Forty-five percent of patients were male. In $21.61 \%$ of patient with gastric outlet obstruction $(n=43)$, the condition was secondary to benign causes. Mean age of patients that underwent the EUS-GE procedure for both benign and malignant gastric outlet obstruction was $64.52 \pm 1.37$ years with a pooled mean follow-up period of $4.32 \pm 1.65$ months. Further baseline study and patient characteristics are highlighted in $>$ Table 1.

\section{Procedure characteristics of included studies}

Both assisted and unassisted EUS-GE techniques were included with an overall mean procedure time for all techniques of 43.49 \pm 19.69 minutes. The most commonly employed technique was direct EUS-GE $(n=134)$, followed by balloon-assisted $(n=35)$, then EPASS $(n=20)$, next ultra-slim $(n=5)$, afterwards nasobiliary drain $(n=3)$, and finally NOTES $(n=2)$. All studies included the CE-LAMS device; however, one study by Chen et al. also utilized the NCE-LAMS in $7.04 \%$ patients $(n=14)$. A total of $99.50 \%$ of patients $(n=198)$ had placement of a size $15 \mathrm{~mm} \times 10 \mathrm{~mm}$ LAMS for EUS-GE, with $10 \mathrm{~mm} \times 10 \mathrm{~mm}$ LAMS placement in only one patient. Additional procedure-specific characteristics are described in $>$ Table $\mathbf{1}$.

\section{Efficacy and clinical effectiveness of EUS-guided gastroenterostomy}

The immediate technical success of EUS-GE as reported in all included studies was $92.90 \%\left(95 \% \mathrm{Cl} ; 88.26\right.$ to $\left.95.79 ; \mathrm{I}^{2}=0.00 \%\right)$ ( $\triangleright$ Fig.2a). Four studies documented clinical success with a pooled success rate of $90.11 \%\left(95 \% \mathrm{Cl} ; 84.64\right.$ to $93.44 ; \mathrm{I}^{2}=$ $0.00 \%$ ) ( Fig. 2b). Clinical success as determined by the GOOSS was only reported in one study [27]. Based upon this study by Itoi et al., the mean post-GOOSS score was significantly higher compared to the pre-GOOSS score $(2.94 \pm 0.23$ versus $0.6 \pm 0.75 ; P<0.001$ ) (Supplemental Fig. 1 ). The median postGOOSS score was significantly higher than the pre-GOOSS score $(0.00$ versus $3.00 ; P<0.001)$.

\section{Safety of EUS-guided gastroenterostomy}

Serious AEs occurred in $5.61 \%\left(95 \% \mathrm{Cl} ; 2.87\right.$ to $10.67 ; \mathrm{I}^{2}=$ $1.67 \%$ ) of cases and were related to peritonitis, perforation, bleeding, and abdominal pain ( $\vee$ Fig. 3a). Overall, the EA rate for the EUS-GE procedure was $10.59 \%$ (95\% Cl; 6.74 to 16.25 ; $\mathrm{I}^{2}=27.17 \%$ ) (Supplemental Fig. 2a). Minor AEs were reported in $5.78 \%\left(95 \% \mathrm{Cl} ; 3.13\right.$ to $\left.10.41 ; \mathrm{I}^{2}=0.00 \%\right)$ of procedures and were most commonly due to stent migration (Supplemental Fig. 2b). Need for repeat procedure or conversion to open surgical intervention was reported in four studies. Repeat intervention was required for $11.43 \%\left(95 \% \mathrm{Cl} ; 7.29-17.46 ; I^{2}=\right.$ $17.38 \%$ ) of EUS-GE procedures ( $\mathbf{F i g . 3 b}$ ).

\section{Risk of bias assessment}

All studies were evaluated using the Newcastle-Ottawa Quality Assessment Scale scores with quality assessment for each study shown in $>$ Table 1. All included studies were considered to be of high quality with scores $\geq 5$. Despite the limited number of included studies, publication bias was assessed [28]. Based on visual inspection of the funnel plot as well as quantitative measurement using the Egger regression test, there was no evidence of publication bias ( $>$ Fig. 4 ).

\section{Discussion}

This systematic review and meta-analysis demonstrates that EUS-GE is a technically feasible and effective endoscopic procedure for management of patients with benign and malignant gastric outlet obstruction. With an immediate technical success rate of $92.90 \%$ and a low $5.61 \%$ rate of serious AEs, EUS-GE appears to be relatively safe and well-tolerated. Given the significant morbidity of surgical gastrojejunostomy and high incidence of recurrent gastric outlet obstruction after endoscopic luminal stenting, EUS-GE shows promise as a fledgling procedure with the potential to become a first-line minimally-invasive therapy for patients with benign and malignant gastric outlet obstruction

\section{Evolution of EUS-GE}

First performed in a porcine animal model by Fritscher-Ravens et al. more than a decade ago, EUS-GE provided a new method for stitching under flexible EUS control but required endoscope exchange and use of special devices, thus limiting adoption in clinical practice $[8,10]$. However, development of a lumen-apposing self-expandable fully covered metal stent (AXIOS Boston Scientific Corp., Marlborough, Massachusetts, United States) able to safely appose two juxtaposed luminal structures and form an endoscopic anastomosis has brought new insights into development of EUS-GE [8, 29].

Although early animal models were successful, translation of these findings in humans has been challenging, represented by identification of the proper distal duodenal or proximal jejunal loop to be accessed from the gastric body to create the anastomosis $[8,30]$. While a variety of EUS-GE techniques are in practice, addition of EUS allows for proper recognition of bowel structure, typically using a 19- to 22-gauge to puncture the loop, followed by contrast injection and guidewire placement $[5,8]$. Next, EUS-GE involves exchange with a CE-LAMS device to cautery puncture and dilate the tract, followed by distal flange deployment into the bowel lumen, withdrawal of the endoscope and the bowel loop toward the gastric cavity where the proximal flange is finally deployed. All included studies in this meta-analysis involved a CE-LAMS to perform EUS-GE, though one study included both CE-LAMS and NCE-LAMS [23].

\section{Current standard of care and other available endoscopic treatments}

Surgical gastrojejunostomy is the current standard of care for treatment of gastric outlet obstruction; however, the significant morbidity and high complication rate associated with surgery has necessitated a search for less invasive and better tolerated alternatives $[6,7,9]$. Endoscopic alternatives including placement of luminal enteral stents have been employed and have achieved comparable technical and clinical success rates with lower overall AE rates as compared to the traditional surgi- 


\begin{tabular}{|c|c|c|c|c|c|c|}
\hline 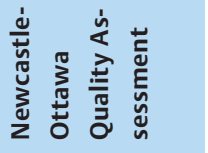 & $\stackrel{\text { Ln }}{\text { ก̊ }}$ & นn & $\stackrel{\circ}{r}$ & $\stackrel{\circ}{r}$ & i̊ & $\stackrel{\circ}{\dot{\varphi}}$ \\
\hline 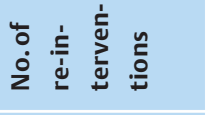 & $\stackrel{\stackrel{n}{N}}{N}$ & $\frac{n}{\infty}$ & $\frac{n}{m}$ & $\frac{\sqrt{n}}{N}$ & 1 & $\stackrel{\stackrel{\circ}{N}}{=}$ \\
\hline 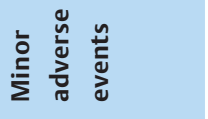 & $\frac{\stackrel{d}{N}}{\sqrt{n}}$ & $\stackrel{n}{=}$ & $\frac{n}{N}$ & $\frac{\mathrm{N}}{\mathrm{N}}$ & $\stackrel{\text { I }}{=}$ & $\frac{n}{N}$ \\
\hline $\begin{array}{l}气 \\
\vdots\end{array}$ & $\frac{d}{m}$ & $\stackrel{\text { 㔯 }}{=}$ & $\stackrel{n}{\frac{n}{r}}$ & $\frac{0}{\mathfrak{N}}$ & $\stackrel{\text { I }}{=}$ & $\frac{\stackrel{0}{N}}{m}$ \\
\hline 宮 厄 & $\stackrel{\frac{\pi}{N}}{\stackrel{N}{N}}$ & $\frac{i n}{i n}$ & $\frac{N}{\stackrel{N}{\infty}}$ & $\frac{\pi}{\stackrel{N}{N}}$ & 1 & 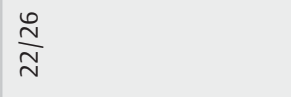 \\
\hline 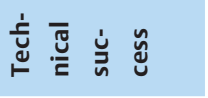 & $\frac{\stackrel{d}{d}}{\frac{d}{d}}$ & $\frac{n}{n}$ & $\frac{n}{g}$ & $\frac{\text { N }}{\stackrel{N}{N}}$ & 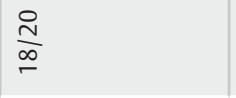 & 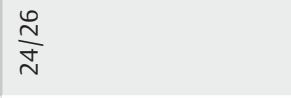 \\
\hline 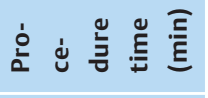 & 1 & m & iे & $\stackrel{\circ}{\infty}$ & $\stackrel{\stackrel{\llcorner}{N}}{\sim}$ & 1 \\
\hline 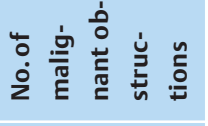 & $\approx$ & $\stackrel{\infty}{+}$ & mे & $\stackrel{\llcorner}{\leftarrow}$ & ì & $\mp$ \\
\hline 总 & 0 & $a$ & $\stackrel{\infty}{\leftarrow}$ & $r$ & 0 & $a$ \\
\hline$\dot{0} \frac{\dot{d}}{\frac{\pi}{\pi}} \frac{\bar{\partial}}{n}$ & $\sigma$ & $\stackrel{\infty}{\sim}$ & $\approx$ & $=$ & $\stackrel{\circ}{\circ}$ & $=$ \\
\hline 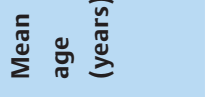 & ه. & $\tilde{\emptyset}$ & $\tilde{\sigma}$ & $\tilde{\sigma}$ & & ڤ̃ \\
\hline 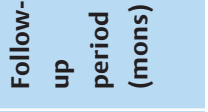 & 1 & ڤn & $\stackrel{\infty}{m}$ & $\stackrel{\infty}{m}$ & $\stackrel{m}{m}$ & $\stackrel{\infty}{-}$ \\
\hline$\dot{\dot{\theta}} \check{2}$ & $\stackrel{\sim}{\sim}$ & in & กิ & $\tilde{N}$ & $\stackrel{\curvearrowright}{N}$ & $\stackrel{\stackrel{\sim}{\sim}}{\sim}$ \\
\hline 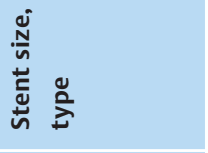 & 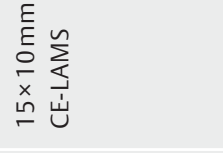 & 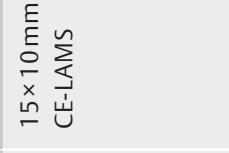 & 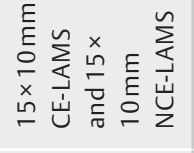 & 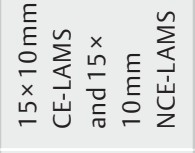 & 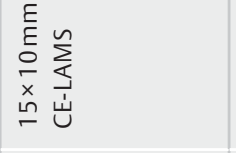 & 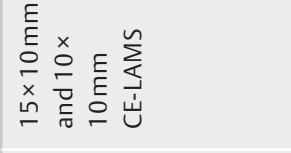 \\
\hline 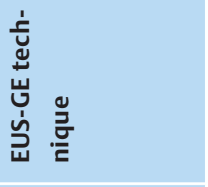 & 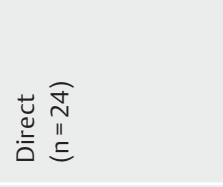 & 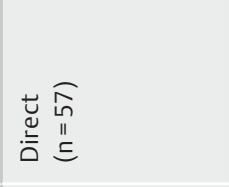 & 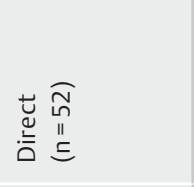 & 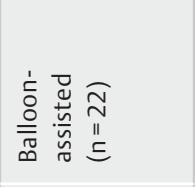 & 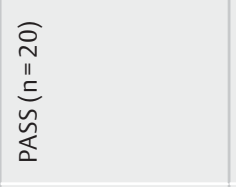 & 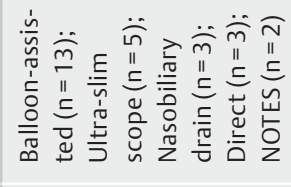 \\
\hline 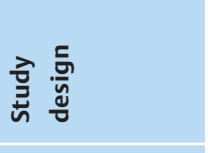 & 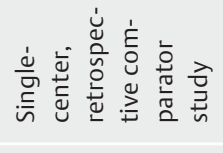 & 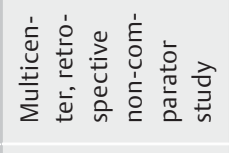 & 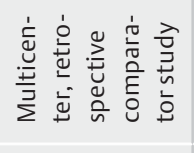 & 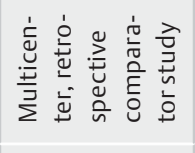 & 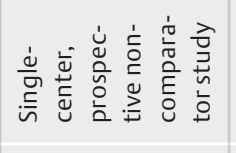 & 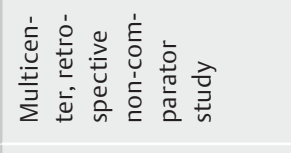 \\
\hline$\stackrel{\text { गू }}{\nu}$ & $\stackrel{\circ}{\stackrel{0}{N}}$ & $\stackrel{\infty}{\stackrel{N}{N}}$ & $\stackrel{\infty}{\stackrel{\infty}{N}}$ & $\stackrel{\infty}{\stackrel{2}{N}}$ & $\stackrel{0}{\stackrel{0}{N}}$ & $\stackrel{0}{\grave{n}}$ \\
\hline 全 & $\begin{array}{l}\frac{\dot{\pi}}{\tilde{\Xi}} \\
\stackrel{\Xi}{\Xi}\end{array}$ & 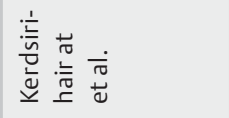 & 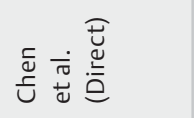 & 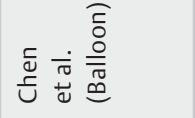 & $\begin{array}{l}\bar{\pi} \\
\stackrel{0}{0} \\
\overline{9} \\
\underline{9}\end{array}$ & 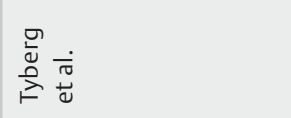 \\
\hline
\end{tabular}


Technical success of EUS-GE

\section{Study name}

Statistics for each study

Event rate

Ge et al. (2019)

Kerdsirihair at et al. (2018)

Chen et al. - Direct (2018)

Chen et al. - Balloon (2018)

Itoi et al. (2016)

Tyberg et al. (2016)

\section{Clinical success of EUS-GE}

Study name

\begin{tabular}{ll} 
& Event rate \\
\hline Ge et al. (2019) & 0.9583 \\
Kerdsirihair at et al. (2018) & 0.8947 \\
Chen et al. - Direct (2018) & 0.9231 \\
Chen et al. - Balloon (2018) & 0.9091 \\
Tyberg et al. (2016) & 0.8462 \\
& 0.9011
\end{tabular}

b

$0.8123 \quad 0.9708$

$\begin{array}{lllll}0.9800 & 0.7487 & 0.9988 & 2.7243 & 0.0064 \\ 0.9298 & 0.8275 & 0.9734 & 4.9834 & 0.0000 \\ 0.9423 & 0.8358 & 0.9813 & 4.6963 & 0.0000 \\ 0.9091 & 0.7004 & 0.9772 & 3.1048 & 0.0019 \\ 0.9000 & 0.6762 & 0.9749 & 2.9479 & 0.0032 \\ 0.9231 & 0.7393 & 0.9807 & 3.3763 & 0.0007 \\ 0.9290 & 0.8826 & 0.9579 & 9.0912 & 0.0000\end{array}$

Statistics for each study

Lower limit Upper limit Z-value $\boldsymbol{P}$-value

$\begin{array}{llll}0.7565 & 0.9942 & 3.0695 & 0.0021\end{array}$

$0.7848 \quad 0.9519$

$0.7004 \quad 0.9772$

$0.6546 \quad 0.9410$

0.8464

0.9377

4.9585

4.7748

3.1048

3.1363

8.6185
Event rate and $95 \% \mathrm{Cl}$

P-value

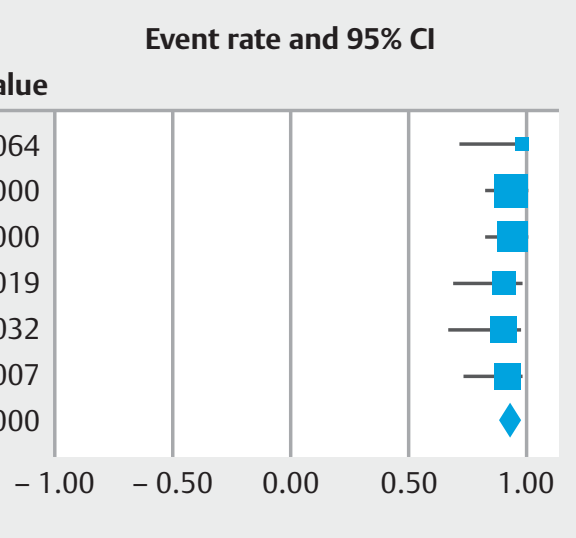

Event rate and $95 \% \mathrm{Cl}$

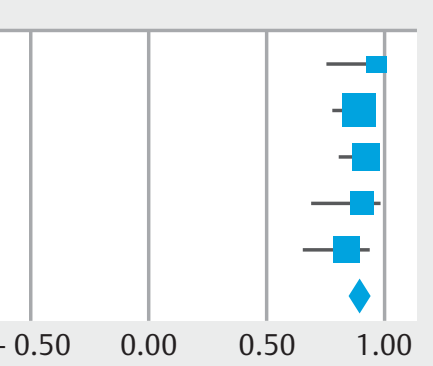

- Fig. 2 a Immediate success rate for EUS-guided gastroenterostomy for the treatment of gastric outlet obstruction. b Clinical success rate for EUS-guided gastroenterostomy for the treatment of gastric outlet obstruction.

cal approach [31]. Nevertheless, enteral stenting provides a less durable option for patients with a prolonged life expectancy, plagued by frequent need for reintervention due to stent migration or stent tumor ingrowth/overgrowth [6, 9, 32].

\section{EUS-GE versus other treatments}

Although evidence is limited, a recent study by Kashhab and colleagues comparing outcomes of EUS-GE to surgical gastrojejunostomy for treatment of malignant gastric outlet obstruction found the technical success rate was significantly higher in the surgical cohort ( $100 \%$ vs. $87 \%, P=0.009$ ) [33]. While the surgical approach was more feasible and less technically challenging, the clinical success rate was no different between the surgical and EUS groups ( $90 \%$ versus $87 \%, P=0.18$ ). There was also a favorable trend with lower recurrence of gastric outlet obstruction in the EUS-GE group (3\%) as compared to the surgical group (14\%); although this was not statistically significant $(P=0.08)$. In another study by Manuel Perez-Miranda et al., technical success was nt different between an EUS-GE cohort and patients undergoing laparoscopic gastrojejunostomy ( $88 \%$ versus $100 \%, P=0.11$ ), although EUS-GE was associated with a significantly lower rate of AEs ( $12 \%$ versus $41 \%, P=0.0386$ ) [34]. Both of these studies were excluded from our systematic review and meta-analysis due to concerns regarding overlap- ping enrollment periods and duplication of patient data [26, 33, 34].

Another study by Chen et al. compared EUS-GE with endoscopic enteral stenting and demonstrated a significantly lower risk of recurrence and reintervention in the EUS-GE group compared to the enteral stenting group ( $4.3 \%$ vs $28.6 \%, P=0.015$ ) [35]. Even on multivariable analysis, enteral stent placement was independently associated with need for reintervention (OR 12.8, $P=0.027$ ). These authors also found similar rates of technical success between the two strategies and a trend towards favorable clinical success among the EUS-GE group. While again not included in our meta-analysis due to concerns regarding overlapping enrollment periods, the results suggest EUS-GE provides longer-lasting luminal patency and reduces occurrence of stent obstruction, with a reasonable procedural risk, without the morbidity associated with a surgical procedure $[8,23,35]$.

\section{Clinical implications and future direction}

Although these early data are promising and suggest that the procedure is safe and effective, there is not yet enough evidence at this time to endorse EUS-GE as the standard of care for gastric outlet obstruction [9]. Future, well-designed RCTs and prospective studies are needed to further validate these findings. Ultimately, more comparator studies are needed to 
Serious adverse event rate of EUS-GE Study name

Statistics for each study

\begin{tabular}{llllll} 
& $\begin{array}{l}\text { Event } \\
\text { rate }\end{array}$ & $\begin{array}{l}\text { Lower } \\
\text { limit }\end{array}$ & $\begin{array}{l}\text { Upper } \\
\text { limit }\end{array}$ & Z-value & P-value \\
\hline Ge et al. (2019) & 0.0833 & 0.0209 & 0.2788 & -3.2468 & 0.0012 \\
Kerdsirihair at et al. (2018) & 0.0175 & 0.0025 & 0.1143 & -3.9899 & 0.0001 \\
Chen et al. - Direct (2018) & 0.0192 & 0.0027 & 0.1243 & -3.8938 & 0.0001 \\
Chen et al. - Balloon (2018) & 0.0217 & 0.0013 & 0.2681 & -2.6623 & 0.0078 \\
Itoi et al. (2016) & 0.0500 & 0.0070 & 0.2822 & -2.8699 & 0.0041 \\
Tyberg et al. (2016) & 0.1154 & 0.0377 & 0.3029 & -3.3182 & 0.0009 \\
& 0.0561 & 0.0287 & 0.1067 & -7.9292 & 0.0000
\end{tabular}

Event rate and $95 \% \mathrm{Cl}$

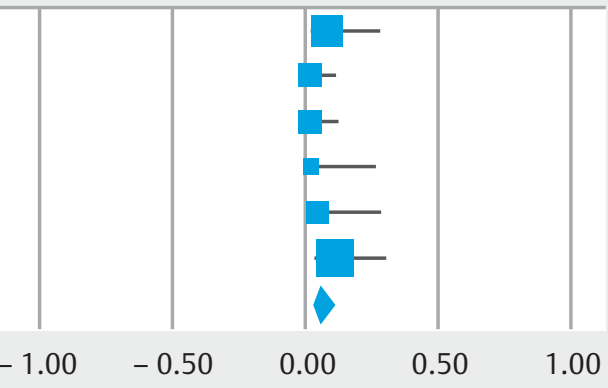

a

Statistics for each study

Study name

b

Ge et al. (2019)

Kerdsirihair at et al. (2018)

Chen et al. - Direct (2018)

Chen et al. - Balloon (2018)

Tyberg et al. (2016)

$\begin{array}{lllll}\begin{array}{l}\text { Event } \\ \text { rate }\end{array} & \begin{array}{l}\text { Lower } \\ \text { limit }\end{array} & \begin{array}{l}\text { Upper } \\ \text { limit }\end{array} & \text { Z-value } & \boldsymbol{P} \text {-value } \\ 0.0800 & 0.0201 & 0.2649 & -3.3130 & 0.0009 \\ 0.1509 & 0.0773 & 0.2738 & -4.5015 & 0.0000 \\ 0.0577 & 0.0187 & 0.1642 & -4.6963 & 0.0000 \\ 0.1818 & 0.0699 & 0.3964 & -2.7210 & 0.0065 \\ 0.0385 & 0.0054 & 0.2279 & -3.1564 & 0.0016 \\ 0.1143 & 0.0729 & 0.1746 & -8.1130 & 0.0000\end{array}$

$\begin{array}{llllll}0.1143 & 0.0729 & 0.1746 & -8.1130 & 0.0000\end{array}$
Event rate and 95\% Cl

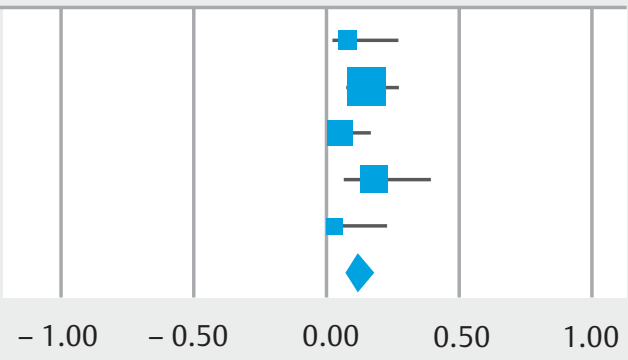

- Fig. 3 a Serious adverse events for EUS-guided gastroenterostomy for the treatment of gastric outlet obstruction. b Repeat interventions required after EUS-guided gastroenterostomy for the treatment of gastric outlet obstruction.

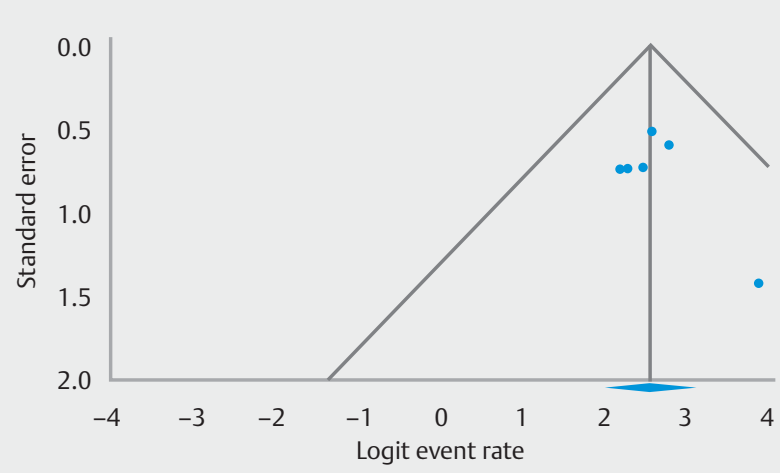

Egger's regression intercept

$\begin{array}{lr}\text { Intercept } & 0.82809 \\ \text { Standard error } & 0.82163 \\ \text { 95 \% lower limit (2-tailed) } & -1.45313 \\ \text { 95\% upper limit (2-tailed) } & 3.10930 \\ \text { t-value } & 1.00786 \\ \text { df } & 4.00000 \\ P \text { value (1-tailed) } & 0.18527 \\ P \text { value (2-tailed) } & 0.37054\end{array}$

Fig. 4 Funnel plot of publication bias and Eggers regression test for included studies to assess EUS-guided gastroenterostomy for the treatment of gastric outlet obstruction. evaluate the efficacy and tolerability of EUS-GE as compared to enteral and surgical gastrojejunostomy as well as a comparison between various EUS-GE techniques and LAMS size. Additional limitations to mainstream use include the notion that the EUSGE procedure is technically demanding, thus at present, it should be performed only by highly trained experts in interventional EUS [8]. It is important to note EUS-GE is not devoid of morbidity and AEs may be significant when they occur. Formal training and improved familiarity with the procedure overall will be required prior to seeing any meaningful clinical implication. Only then will the procedure likely see adoption into clinical practice.

\section{Strengths and limitations}

Specific limitations to this study include reliance largely on retrospectively collected data, differences in patient population across studies, lack of long-term follow-up periods, and inclusion of multiple EUS-GE techniques. While important to consider, heterogeneity of studies was low based on our analyses. In addition, while clinical success as determined by authors was reported in all studies, objective measures of clinical success (i.e. the GOOSS) were only reported in one study [27]. As the evidence stands now, there remains limited data regarding EUS-GE with sparse literature and numerous studies reporting 
redundant patient information. Several studies in our literature search were excluded due to concerns regarding overlapping results from the same cohort of EUS-GE individuals. Although publication bias is not typically assessed with funnel plot asymmetry with fewer than eight to 10 studies in a meta-analysis, qualitative and quantitative publication bias was performed as pooled results of uncontrolled, retrospective studies carry a high risk of amplifying selection bias [28]. Despite this concern, our meta-analysis demonstrated no evidence of publication bias.

In addition, there remains a lack of data to compare EUS-GE directly with surgical gastrojejunostomy with only one study demonstrating similar efficacy among more complex patients [34]. With only five studies, subgroup analysis based upon EUS-GE technique or benign and malignant causes of gastric outlet obstruction, both of which would be highly clinically relevant, was not possible. An additional concern with any endoscopic procedure or technique is the learning curve or clinical expertise needed to perform an effective procedure [16]. It is possible the technical success achieved in these studies may be not be generalizable to centers with less familiarity or proven expertise.

Despite these limitations, this study has several strengths. Most importantly, our meta-analysis methodologically summarizes all available data to evaluate the feasibility, efficacy, and tolerability of the EUS-GE procedure. Overall, EUS-GE was demonstrated to be an effective device for gastric outlet obstruction in both short- and longer-term follow-up with impressive technical and clinical success rates and few serious AEs. Although we were unable to provide a subgroup analysis based upon gastric outlet obstruction etiology and EUS-GE technique, these findings provide an important step forward in proving the effectiveness and utility of further trials. With a relatively even distribution of benign and malignant gastric outlet obstructions included in this analysis, it is reasonable to assume EUS-GE is an effective modality for both etiologies. These data are pivotal to improve both symptom management and quality of life for individuals with gastric outlet obstruction, regardless of etiology, and may suggest an increasing role for EUS-GE.

\section{Conclusions}

Based upon our systematic review and meta-analysis, EUS-GE appears to be an effective and safe minimally invasive alternative for treatment of benign and malignant gastric outlet obstruction. Given the significant morbidity associated with surgical gastrojejunostomy reaching nearly $40 \%$, less invasive options that provide durable and effective results will continue to be sought. Although EUS-GE remains a technically challenging procedure with limited evidence to date, it demonstrated high immediate technical and clinical success rates of $92.90 \%$ and $90.11 \%$, respectively, with a low serious AE profile of $5.61 \%$. While surgical gastrojejunostomy will continue to be the standard of care, this systematic review and meta-analysis suggests an increased role for EUS-GE in management of gastric outlet obstruction.

\section{Competing interests}

Dr. Thompson is a consultant for Boston Scientific, Olympus, and Apollo Endosurgery. Dr. Rustagi is a consultant for Boston Scientific and Cook Endoscopy.

\section{References}

[1] Chowdhury A, Dhali GK, Banerjee PK. Etiology of gastric outlet obstruction. Am J Gastroenterol 1996; 91: 1679

[2] Johnson CD. Gastric outlet obstruction malignant until proved otherwise. Am J Gastroenterol 1995; 90: 1740

[3] Johnson CD, Ellis H. Gastric outlet obstruction now predicts malignancy. Br J Surg 1990; 77: 1023 - 1024

[4] Shone DN, Nikoomanesh P, Smith-Meek MM et al. Malignancy is the most common cause of gastric outlet obstruction in the era of $\mathrm{H} 2$ blockers. Am J Gastroenterol 1995; 90: 1769-1770

[5] Storm AC, Ryou M. Advances in the endoscopic management of gastric outflow disorders. Curr Opin Gastroenterol 2017; 33: 455-460

[6] Khashab M, Alawad AS, Shin E] et al. Enteral stenting versus gastrojejunostomy for palliation of malignant gastric outlet obstruction. Surg Endosc 2013; 27: 2068-2075

[7] Itoi T, Baron TH, Khashab MA et al. Technical review of endoscopic ultrasonography-guided gastroenterostomy in 2017. Dig Endosc 2017; 29: $495-502$

[8] Rimbas M, Larghi A, Costamagna G. Endoscopic ultrasound-guided gastroenterostomy: Are we ready for prime time? Endosc Ultrasound 2017; 6: 235-240

[9] Amin S, Sethi A. Endoscopic ultrasound-guided gastrojejunostomy. Gastrointest Endosc Clin N Am 2017; 27: 707 - 713

[10] Fritscher-Ravens A, Mosse CA, Mills TN et al. A through-the-scope device for suturing and tissue approximation under EUS control. Gastrointest Endosc 2002; 56: 737-742

[11] Liberati A, Altman DG, Tetzlaff J et al. The PRISMA statement for reporting systematic reviews and meta-analyses of studies that evaluate health care interventions: explanation and elaboration. Annals Internal Med 2009; 151: W65-W94

[12] Stroup DF, Berlin JA, Morton SC et al. Meta-analysis of observational studies in epidemiology: a proposal for reporting. Meta-analysis Of Observational Studies in Epidemiology (MOOSE) group. JAMA 2000; 283: $2008-2012$

[13] Adler DG, Baron TH. Endoscopic palliation of malignant gastric outlet obstruction using self-expanding metal stents: experience in $36 \mathrm{pa}-$ tients. Am J Gastroenterol 2002; 97: 72-78

[14] DerSimonian R, Laird N. Meta-analysis in clinical trials. Controlled Clin Trials 1986; 7: $177-188$

[15] Stuart A, Ord JK. Kendall's Advanced Theory of Statistics. 6th ed. London: Edward Arnold; 1994

[16] McCarty TR, Itidiare M, Njei B et al. Efficacy of transoral incisionless fundoplication for refractory gastroesophageal reflux disease: a systematic review and meta-analysis. Endoscopy 2018; 50: 708-725

[17] McCarty TR, Njei B. Self-expanding metal stents for acute refractory esophageal variceal bleeding: A systematic review and meta-analysis. Dig Endosc 2016; 28: 539- 547

[18] Wells G, Shea B et al. The Newcastle-Ottawa Scale (NOS) for assessing the quality of nonrandomised studies in meta-analysis. 3rd Symposium on Systematic Reviews: Beyond the Basics; July 3-5; Oxford. 2000. Available at: http://www.ohri.ca/programs/clinical_epidemiology/oxford.asp [Accessed 11 April 2019] 
[19] Jadad AR, Moore RA, Carroll D et al. Assessing the quality of reports of randomized clinical trials: is blinding necessary? Control Clin Trials 1996; 17: $1-12$

[20] Higgins JP, Thompson SG, Deeks JJ et al. Measuring inconsistency in meta-analyses. BMJ 2003; 327: 557 - 560

[21] Egger M, Davey Smith G et al. Bias in meta-analysis detected by a simple, graphical test. BMJ 1997; 315: 629-634

[22] Easterbrook PJ, Berlin JA, Gopalan R et al. Publication bias in clinical research. Lancet 1991; 337: 867-872

[23] Chen YI, Kunda R, Storm AC et al. EUS-guided gastroenterostomy: a multicenter study comparing the direct and balloon-assisted techniques. Gastrointest Endosc 2018; 87: 1215-1221

[24] Ge PS, Young JY, Dong W et al. EUS-guided gastroenterostomy versus enteral stent placement for palliation of malignant gastric outlet obstruction. Surg Endosc 2019: doi:10.1007/s00464-018-06636-3 [Epub ahead of print]

[25] Kerdsirichairat T, Irani S, Yang J et al. Durability and long-term outcomes of direct EUS-guided gastroenterostomy using lumen-apposing metal stents for gastric outlet obstruction. Endosc Int Open 2019; 7: E144-E150

[26] Tyberg A, Perez-Miranda M, Sanchez-Ocana R et al. Endoscopic ultrasound-guided gastrojejunostomy with a lumen-apposing metal stent: a multicenter, international experience. Endosc Int Open 2016; 4: E276-E281

[27] Itoi T, Ishii K, Ikeuchi $\mathrm{N}$ et al. Prospective evaluation of endoscopic ultrasonography-guided double-balloon-occluded gastrojejunostomy bypass (EPASS) for malignant gastric outlet obstruction. Gut 2016; 65: $193-195$
[28] Higgins JP, Green S. Cochrane Handbook for Systematic Reviews of Interventions. Version 5.1.0. Available from: http://handbook-5-1. cochrane.org [Accessed 9 April 2019]

[29] Itoi T, Binmoeller KF, Shah J et al. Clinical evaluation of a novel lumenapposing metal stent for endosonography-guided pancreatic pseudocyst and gallbladder drainage (with videos). Gastrointest Endosc 2012; 75: $870-876$

[30] Binmoeller KF, Shah JN. Endoscopic ultrasound-guided gastroenterostomy using novel tools designed for transluminal therapy: a porcine study. Endoscopy 2012; 44: 499-503

[31] Jeurnink SM, van Eijck CH, Steyerberg EW et al. Stent versus gastrojejunostomy for the palliation of gastric outlet obstruction: a systematic review. BMC Gastroenterol 2007; 7: 18

[32] Jeurnink SM, Steyerberg EW, van Hooft JE et al. Surgical gastrojejunostomy or endoscopic stent placement for the palliation of malignant gastric outlet obstruction (SUSTENT study): a multicenter randomized trial. Gastrointest Endosc 2010; 71: 490 - 499

[33] Khashab MA, Bukhari M, Baron TH et al. International multicenter comparative trial of endoscopic ultrasonography-guided gastroenterostomy versus surgical gastrojejunostomy for the treatment of malignant gastric outlet obstruction. Endosc Int Open 2017; 5: E275-E281

[34] Perez-Miranda M, Tyberg A, Poletto D et al. EUS-guided gastrojejunostomy versus laparoscopic gastrojejunostomy: an international collaborative study. J Clin Gastroenterol 2017; 51: 896 - 899

[35] Chen YI, Itoi T, Baron TH et al. EUS-guided gastroenterostomy is comparable to enteral stenting with fewer re-interventions in malignant gastric outlet obstruction. Surg Endosc 2017; 31: 2946-2952 